The Bulletin of Symbolic Logid

Volume 9, Number 3, Sept. 2003

\title{
IN MEMORIAM: WALTER FELSCHER
}

1931-2000

Walter Felscher was born on October 12, 1931, in Brandenburg and died on December 12, 2000. He studied mathematics at the Free University of Berlin and received his doctoral degree in 1956 under F. W. Levi. He became a lecturer at the University of Freiburg where he was qualified as an academic teacher. He held a research position at Dalhousie University from 1969 to 1971. In 1971 he was appointed to the chair of "Logic, Foundations and History of Mathematics" at the University of Tübingen. He held this position, first in the Mathematics Department and later in the Department of Computer Science, until his retirement.

Walter Felscher truly was one of the outstanding logicians in Germany with highly diversified activities in logic. An indication of his reputation is the fact that he chaired or co-chaired no less than twenty-one conferences on logic, set theory and universal algebra at the Mathematics Institute of Oberwolfach. Much of his influence was on a personal basis; he corresponded widely and actively with mathematical logicians and philosophers throughout his life.

His scientific work reflects many aspects of mathematical logic and underwent several changes: universal algebra and category theory, set and model theory, and finally proof theory and the logic of arithmetic. One can, however, identify an underlying theme: Mathematical logic is treated as a mathematical discipline and mathematical, in particular algebraic methods play a dominant role. In this respect a major thread in his work can be described as the emphasis of the adjective "mathematical" in mathematical logic, or more precisely, the application of algebraic methods to logic, for which a few examples will be given.

The lines of argument already become apparent in his 1965 work on "Adjungierte Funktoren und primitive Klassen". It revolves around Birkhoff's theorem that characterizes primitive classes of algebras as those that are equationally definable. The abstract setting in terms of category theory allows, however, to cover a broad area of variations and related results. For example, the compactness theorems for equality logic and quantifier-free logic are among the consequences. The algebraic view of logic was extended in the paper "An Algebraic Approach to First Order Logic" where all relevant concepts were formulated in a strictly algebraic way, hence allowing one to apply the machinery of universal algebra and category theory. 
A question open for several years was concerned with intuitionistic logic. The problem was the equivalence of dialogue strategies and more familiar proof systems, like Gentzen's calculus LJ; Felscher's solution employed syntactic transformations between the different calculi.

Much of his work is published in his various books, on "Naïve Set Theory", "Recursive Functions" and "Mathematical Logic". In the first books another aspect is worth mentioning: historical precision. I remember a typical episode: The two editions of Dedekind's book "Was sind und was sollen die Zahlen?" differed in some respects; the second one contained a passage which could have been copied by Dedekind from Bolzano. Walter Felscher went to the Mathematics library in Braunschweig and looked in the archives of the 19th century and it was in fact recorded that Dedekind had read the relevant writing of Bolzano at the time in question.

Walter Felscher's main heritage is surely the three volumes of "Lectures on Mathematical Logic" which appeared in 2000. They contain a complete and admirably concise view of logic, with respect to both technical detail and historical comment. These books stem from his lectures in the early sixties, which I had the opportunity and privilege to attend. I firmly believe that in particular these last books will have a continuing influence on future generations of logicians.

MiCHAEL RichteR 